\title{
Cluster-resolved dynamic scaling theory and universal corrections for transport on percolating systems
}

\author{
Axel Kammerer, Felix Höfling and Thomas Franosch \\ Arnold Sommerfeld Center for Theoretical Physics (ASC) and Center for NanoScience (CeNS), \\ Fakultät für Physik, Ludwig-Maximilians-Universität München, Theresienstraße 37, 80333 München, Germany
}

PACS 64.60.ah - General studies of phase transitions: Percolation

PACS 05.10.-a - Computational methods in statistical physics and nonlinear dynamics

\begin{abstract}
For percolating systems, we propose a universal exponent relation connecting the leading corrections to scaling of the cluster size distribution with the dynamic corrections to the asymptotic transport behaviour at criticality. Our derivation is based on a cluster-resolved scaling theory unifying the scaling of both the cluster size distribution and the dynamics of a random walker. We corroborate our theoretical approach by extensive simulations for a site percolating square lattice and numerically determine both the static and dynamic correction exponents.
\end{abstract}

Anomalous, subdiffusive transport has been widely observed in biological systems, e.g., in the cytoplasm [1] and in cell membranes [2]. These findings are attributed to an obstructed motion due to the crowded nature of cellular environments, and they have been related to the subdiffusive motion established for percolating systems [3]. The experiments have yielded a wide spectrum of almost con- tinuously changing exponents, but a mechanism generating such a continuum of fractal exponents has not been identified yet. Although the observation windows covered between one and three decades in time, an alternative interpretation in terms of apparent power laws resulting from crossover phenomenona over large windows is conceivable.

For a continuum percolation model, it has been shown recently that the crossover from pure subdiffusion to normal diffusion extends over five decades in time [4]; in addition, the asymptotic behaviour is slowly approached and the large corrections cannot simply be ignored. Thus, it is of general interest to develop a systematic description of universal corrections to scaling in percolating systems.

In this Letter, we generalise the dynamic scaling theory for transport on percolation clusters $[5,6]$ to include the leading corrections. We derive a new exponent relation for dynamic corrections that are inherited from the static structure. Let us start by introducing some notation and a summary of our main results.

First, the cluster size distribution in the infinite lattice becomes fractal, $n_{s} \sim s^{-\tau}$, directly at the percolation threshold $p_{c}$ for large clusters, $s \rightarrow \infty$. The Fisher exponent $\tau$ can be related to the fractal dimension $d_{\mathrm{f}}$ of the infinite cluster via $\tau=1+d / d_{\mathrm{f}}$, provided the space dimension $d$ is smaller than the upper critical dimension $d_{c}=6$. The deviations from the power law for $n_{s}$ are to leading order again determined by a power law

$$
n_{s}\left(p_{c}\right) \simeq A s^{-\tau}\left(1+B s^{-\Omega}\right) \text { for } s \rightarrow \infty .
$$

The correction exponent $\Omega>0$ is by renormalisation group arguments expected to be again universal, i.e., it depends only on the dimension of the system, but not on the details of the lattice. Its role is to quantify how fast the critical manifold is approached and microscopic details become irrelevant. We have determined the value of the correction exponent to $\Omega=0.77 \pm 0.04$ by extensive computer simulations for a two-dimensional (2D) square lattice.

Second, transport of a random walker on the incipient infinite cluster at $p=p_{c}$ is anomalous, quantified by the power-law dependence of the mean-square displacement $\delta r_{\infty}^{2}(t) \sim t^{2 / d_{\mathrm{w}}}$ for large times $t \rightarrow \infty$, where $d_{\mathrm{w}}$ denotes the walk dimension. The approach to this power law is also non-analytic,

$$
\delta r_{\infty}^{2}(t) \simeq A_{\infty} t^{2 / d_{\mathrm{w}}}\left(1+C_{\infty} t^{-y}\right) \quad \text { for } \quad t \rightarrow \infty,
$$

giving rise to the dynamic correction exponent $y>0$.

We shall introduce a cluster-resolved scaling theory for a generalised probability distribution incorporating the 


\begin{tabular}{ccc|l}
\hline \hline$d$ & 2 & \multicolumn{2}{c}{3} \\
\hline$d_{\mathrm{f}}$ & $91 / 48^{\mathrm{a}}$ & \multicolumn{2}{c}{$2.530(4)^{\mathrm{c}}$} \\
$\nu$ & $4 / 3^{\mathrm{a}}$ & \multicolumn{2}{c}{$0.875(1)^{\mathrm{b}}$} \\
$\boldsymbol{\Omega}$ & $\mathbf{0 . 7 7 ( 4 ) ^ { \mathrm { e } }}$ & \multicolumn{2}{c}{$0.64(2)^{\mathrm{b}}$} \\
\hline \multicolumn{3}{c}{ lattice } & \multicolumn{1}{c}{ continuum $^{\mathrm{a}}$} \\
\hline$d_{\mathrm{w}}$ & $2.878(1)^{\mathrm{a}}$ & $3.88(3)^{\mathrm{a}}$ & \multicolumn{1}{c}{$4.81(2)^{\mathrm{d}}$} \\
$z$ & $3.036(1)$ & $5.07(6)$ & $6.30(3)$ \\
$\boldsymbol{y}$ & $\mathbf{0 . 4 9 ( 3 ) ^ { \mathrm { e } }}$ & $\mathbf{0 . 4 2}(\mathbf{2})^{\mathrm{e}}$ & $\mathbf{0 . 3 4}(\mathbf{2})^{\mathrm{e}}$ \\
\hline \hline
\end{tabular}

Table 1: Static and dynamic exponents for the leading and subleading critical behaviour. The uncertainty in the last digit is indicated in parentheses. Sources: (a) ref. [7], (b) ref. [8], (c) calculated from $\tau=2.186(2)$ [9], (d) continuum percolation theory yields $d_{\mathrm{w}}=d_{\mathrm{f}}+2 / \nu[4,10]$, and (e) this work. The dynamic universality class does not split for $d=2[10,11]$. The values for $z$ are derived from an exponent relation; the predictions for $y$ are based on our proposed relation, eq. (3).

static and dynamic properties and derive the new exponent relation

$$
y d_{\mathrm{w}}=\Omega d_{\mathrm{f}} .
$$

By computer simulations, we demonstrate that the assumptions underlying our scaling hypothesis are indeed fulfilled and that the measured correction exponents are compatible with the relation above. We have provided evidence for eq. (3) for $d=3$ before [4], and we will test our theory here for $d=2$; universality allows to choose the simple square lattice for this purpose. In Table 1, we have compiled some critical exponents for transport on percolation clusters in two and three dimensions along with the predictions from this work.

Scaling of the cluster size distribution. - As the probability $p$ for a site to be occupied approaches a certain critical value $p_{c}$, clusters of all sizes emerge and the system becomes self-similar. In the infinite lattice, this is reflected by the power law tail of the distribution $n_{s} \sim s^{-\tau}$; in a finite lattice of box length $L$, the largest cluster is expected to contain of the order of $L^{d_{\mathrm{f}}}$ sites. Off the percolation threshold, the linear extension of the largest finite cluster $\xi$, also referred to as correlation length, diverges in the infinite lattice, $\xi \sim|\epsilon|^{-\nu}$, where $\epsilon:=\left(p-p_{c}\right) / p_{c}$ denotes the reduced distance to the critical point. We now introduce a scaling assumption for $n_{s}$ that encompasses all the singularities as $s \rightarrow \infty, \epsilon \rightarrow 0$, and $L \rightarrow \infty$, including also an irrelevant scaling field $u$,

$$
n_{s}(\varepsilon, L)=L^{-d-d_{\mathrm{f}}} \mathrm{N}\left(s L^{-d_{\mathrm{f}}}, \varepsilon L^{1 / \nu}, u L^{-\omega}\right) .
$$

The first scaling variable $s L^{-d_{\mathrm{f}}}$ states that the size $s$ of a cluster should be compared with the size $L^{d_{\mathrm{f}}}$ of the largest cluster in the box. The second argument $\varepsilon L^{1 / \nu}$ suggests that the system appears similar upon zooming in, $L \mapsto \lambda L$, provided the correlation length is rescaled accordingly, $\xi \mapsto \lambda \xi$. The scaling field $u$ is assumed to

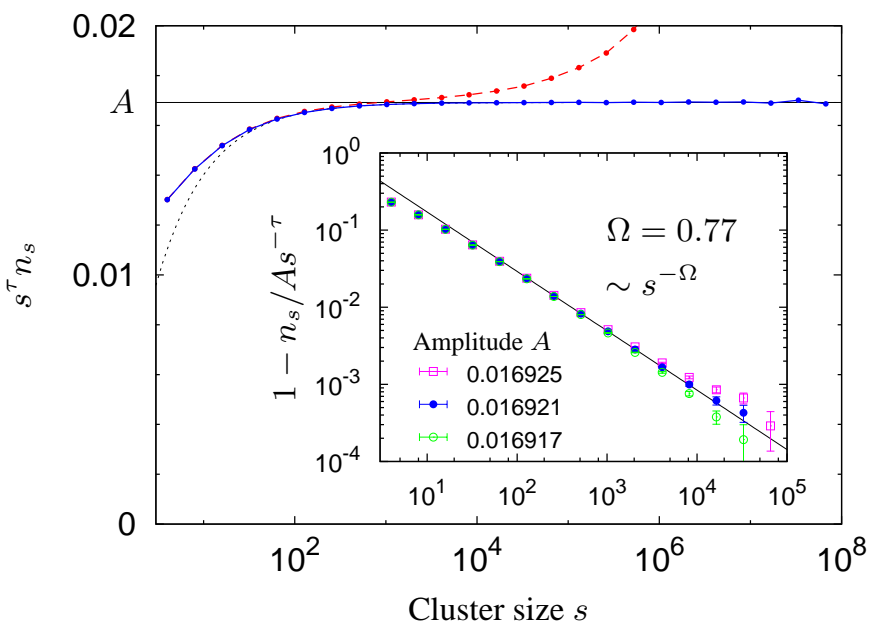

Fig. 1: Rectification of the cluster size distribution $n_{s}$ with the critical power law $s^{-\tau}$ yields the amplitude $A$. Periodic (solid, blue) and free (broken, red) boundary conditions are compared. The dotted line indicates the leading correction to the asymptotic scaling, $n_{s} \simeq A s^{-\tau}\left(1+B s^{-\Omega}\right)$. Inset: the deviation from the asymptotic law on a double-logarithmic plot follows a power law again with the universal correction exponent $\Omega=0.77$.

be the leading irrelevant variable in the renormalisation group sense [12], encoding how some microscopic details of the system fade out as the critical point is approached. Since here the system is finite, $n_{s}$ is an analytic function of all its arguments, and since the arguments $(s, \varepsilon, u)$ appear linearly inside the scaling function $\mathrm{N}$, the same property holds for N. The leading and next-to-leading behaviour is extracted by expanding $\mathrm{N}$ to first order in the irrelevant scaling field $u$,

$$
\begin{aligned}
n_{s}(\varepsilon, L)=L^{-d-d_{\mathrm{f}}} \mathrm{N}_{0}\left(s L^{-d_{\mathrm{f}}}, \varepsilon L^{1 / \nu}\right) & \\
& +L^{-d-d_{\mathrm{f}}-\omega} \mathrm{N}_{1}\left(s L^{-d_{\mathrm{f}}}, \varepsilon L^{1 / \nu}\right),
\end{aligned}
$$

with new analytic functions $\mathrm{N}_{0}, \mathrm{~N}_{1}$, and a factor $u$ has been absorbed in $\mathrm{N}_{1}$. A similar form of eq. (5) was already given by Margolina et al. [13].

Static corrections to scaling. - For large systems, $L \gg \xi$, the dependence on the box size may be eliminated, and eq. (5) for the cluster size distribution can then be written as

$$
n_{s}(\varepsilon)=s^{-\tau} \mathcal{N}_{0}^{ \pm}\left(s \xi^{-d_{\mathrm{f}}}\right)\left[1+s^{-\Omega} \mathcal{N}_{1}^{ \pm}\left(s \xi^{-d_{\mathrm{f}}}\right)\right]
$$

with new one-parameter scaling functions $\mathcal{N}_{0}^{ \pm}(\cdot)$ for the leading behaviour; the superscript \pm distinguishes the $\operatorname{sign}$ of $\varepsilon$. The correction is parametrised in terms of $\mathcal{N}_{1}^{ \pm}(\cdot)$ and the new correction-to-scaling exponent $\Omega:=$ $\omega / d_{\mathrm{f}}$. Specified to the critical point, this yields $n_{s}(\varepsilon=$ $0) \simeq A s^{-\tau}\left(1+B s^{-\Omega}\right)$, with the non-universal amplitudes $A:=\mathcal{N}_{0}^{ \pm}(0)$ and $B:=\mathcal{N}_{1}^{ \pm}(0)$.

A measurement of the correction exponent $\Omega$ requires high quality data for the cluster size distribution $n_{s}$. The 
deviation from leading order has to show an extremely small signal-to-noise ratio in order to follow the power law sufficiently long. This implies a precise knowledge about the leading order, $n_{s} \simeq A s^{-\tau}$, including the prefactor $A$. We have simulated 169,000 square lattices at the threshold $11 p_{c}=0.592746$ [14], using the multi-labelling algorithm by Hoshen and Kopelman [15]. We have used periodic boundary conditions and large lattices with a linear extend of $L=45,000$ sites to minimise the effects due to a finite simulation box [16]. Figure 1 shows a rectification of the obtained distribution $n_{s}$ : the critical power law is followed over more than five decades in $s$. At small cluster sizes, the critical law is approached with another power law, $s^{-\Omega}$, as expected. The figure also displays results for free boundary conditions, where pronounced finite-size effects, however, render a precise observation of the leading critical behaviour nearly impossible.

In two dimensions, we take advantage of the Fisher exponent being known exactly, $\tau=187 / 91$. We have determined the amplitude of the leading order to $A=$ $0.016921(4)$ by linear regression over various intervals in $10^{4} \leq s \leq 10^{7}$. Over these three decades, our data deviate from the leading power law by less than $1 \%$. The deviation from the asymptotic law is shown in the inset of fig. 1 for smaller cluster sizes, $s<10^{5}$. On the double logarithmic plot, the data nicely follow a straight line again over more than two decades in the cluster size. But even with such accurate data, the precise extraction of the exponent is quite intricate. Since we need an asymptotic fit for large (but not too large) $s$, we varied the fit interval for the linear regression in $10^{2} \leq s \leq 10^{4}$; this procedure, however, seems to underestimate the exponent slightly in our case. A more reliable approach for an asymptotic fit is to do another rectification, now of the correction: the best convergence of $s^{\Omega}\left(n_{s} / A s^{-\tau}-1\right) \rightarrow B$ was found for $\Omega=0.77$ with the above value of $A$, yielding $B=-1.01$. The result slightly depends on $A$; varying the amplitude within the above uncertainty, we have obtained error bounds on the correction exponent, $\Omega=0.77 \pm 0.04$.

Universality of $\Omega$ suggests it is independent of the lattice. Indeed, our result is fairly compatible with the result for the triangular lattice [17], where $\Omega$ was found to be between 0.71 and 0.74 ; we attribute the discrepancy to the limited observation window for the correction, $s<10^{3}$, 22 years ago.

Dynamics of a random walker. - Transport on percolating systems is expected to be anomalous since a random walker ("the ant") has to explore the self-similar structures of the ramified clusters. The frozen disorder leads to subdiffusive motion on the infinite cluster directly at the percolation threshold. An independent critical exponent $d_{\mathrm{w}}>2$, referred to as walk dimension, characterises the restricted mean-square displacement (MSD) de-

\footnotetext{
${ }^{1}$ The simulations required a total computation time of 90 hours on 100 cores of the AMD Opteron $2852.6 \mathrm{GHz}$ processor with $2 \mathrm{~GB}$ of RAM per core, which limited the size of the lattices.
}

fined as $\delta r_{\infty}^{2}(t):=\left\langle[\boldsymbol{R}(t)-\boldsymbol{R}(0)]^{2} \mid \boldsymbol{R}(0) \in \mathcal{C}_{\infty}\right\rangle \sim t^{2 / d_{\mathrm{w}}}$, where the average is taken over different realisations of the disorder and $\boldsymbol{R}(t)$ denotes the trajectory of the ant; some values of $d_{\mathrm{w}}$ are listed in Table 1. If walkers on all clusters are considered, the exponent is modified since the average of clusters of all sizes is weighted with the fractal cluster size distribution [18]; the unrestricted MSD follows $\delta r_{\mathrm{av}}^{2}(t) \sim t^{2 / z}$ with the dynamic exponent $z:=2 d_{\mathrm{w}} /\left(2+d_{\mathrm{f}}-d\right)>d_{\mathrm{w}}$. Away from the critical point, the long-time behaviour is either diffusive, $\delta r_{\mathrm{av}}^{2}(t) \simeq 2 d D t$, for $p>p_{c}$ or localised, $\delta r_{\mathrm{av}}^{2}(t \rightarrow \infty)=\ell^{2}$, below the transition, $p<p_{c}$. The diffusion coefficient $D$ and the localisation length $\ell$ exhibit power law behaviour in $\epsilon$.

Cluster-resolved scaling. The statistical information of the transport dynamics is encoded in the van Hove selfcorrelation function $G(\boldsymbol{r}, t):=\langle\delta(\boldsymbol{R}(t)-\boldsymbol{R}(0)-\boldsymbol{r})\rangle$, i.e., the probability that the ant has been displaced by a vector $\boldsymbol{r}$ in time $t$. Here we suggest a cluster-resolved scaling theory for the van Hove function including the leading correction to scaling; in particular, we will derive a relation between static and dynamic exponents. The principle idea is that at the critical point all clusters resemble each other up to proper rescaling and therefore induce similar dynamics after readjusting the time scale. To take advantage of the self-similarity, we generalise the van Hove function to also resolve the cluster size,

$$
G_{s}(\boldsymbol{r}, t):=\left\langle\delta(\boldsymbol{R}(t)-\boldsymbol{R}(0)-\boldsymbol{r}) \mid \boldsymbol{R}(0) \epsilon \mathcal{C}_{s}\right\rangle,
$$

where $\mathcal{C}_{s}$ denotes a cluster of size $s \leq \infty$. After averaging, the information on the detailed structure of the cluster is lost, and we keep only its size as indicator for the dynamics. The ant is trapped for finite $s$, whereas for $s=\infty$ the infinite cluster allows for long-range transport. Let us introduce also the joint probability $P_{s}$ that the ant is on a cluster of size $s$ and has moved a vector $r$ in a given time $t$, as well as a corresponding probability $P_{\infty}$ for the infinite cluster [19],

$$
\begin{aligned}
P_{s}(\boldsymbol{r}, t ; \varepsilon) & :=s n_{s}(\varepsilon) G_{s}(\boldsymbol{r}, t ; \varepsilon), \\
P_{\infty}(\boldsymbol{r}, t ; \varepsilon) & :=P(\varepsilon) G_{\infty}(\boldsymbol{r}, t ; \varepsilon) ;
\end{aligned}
$$

$P(\varepsilon)$ is the probability that a given occupied site belongs to the infinite cluster. We now postulate a scaling relation for the joint probabilities in the same spirit as for eq. (4),

$$
\begin{aligned}
& P_{s}(\boldsymbol{r}, t ; \varepsilon, L)= \\
& L^{-2 d} \mathrm{P}_{\mathrm{F}}\left(r / L, t L^{-d_{\mathrm{w}}}, s L^{-d_{\mathrm{f}}}, \epsilon L^{1 / \nu}, u L^{-\omega}\right), \\
& P_{\infty}(\boldsymbol{r}, t ; \varepsilon, L)= \\
& L^{d_{\mathrm{f}}-2 d} \mathrm{P}_{\infty}\left(r / L, t L^{-d_{\mathrm{w}}}, \epsilon L^{1 / \nu}, u L^{-\omega}\right),
\end{aligned}
$$

where the finite size $L$ of the system has been explicitly considered. (For a finite system, the infinite cluster is defined as the largest cluster of a given percolating lattice.) We have assumed that the leading irrelevant scaling variable $u$ is the same as in the static case. Since the system 
is finite, $\mathrm{P}_{\mathrm{F}}$ and $\mathrm{P}_{\infty}$ are analytic functions in all their arguments. The choice of the arguments is motivated by the well-known scaling relations for the linear extent of walks (exponent $d_{\mathrm{w}}$ ), the cluster size $\left(d_{\mathrm{f}}\right)$, and the correlation length $(\nu)$. The prefactor ensures consistency with the scaling form for the cluster size distribution, eq. (4). Since the van Hove functions $G_{s}$ are normalised, $s n_{s}$ is obtained by integrating eq. (8a) over all $\boldsymbol{r}$, compatible with the scaling hypothesis for $n_{s}$. Integration of eq. 9b yields the familiar result for the strength of the infinite cluster, $P \sim|\epsilon|^{\beta}$, with exponent $\beta=\nu\left(d-d_{\mathrm{f}}\right)$ as $L \rightarrow \infty$. To simplify notation, we suppress the dependence of the scaling functions on $u$ in the remainder of this section.

The limit of infinite system size eliminates the dependence on $L$,

$$
\begin{aligned}
P_{s}(\boldsymbol{r}, t ; \varepsilon) & =R_{s}^{-2 d} \mathcal{P}_{\mathrm{F}}\left(r / R_{s}, t R_{s}^{-d_{\mathrm{w}}}, \varepsilon R_{s}^{1 / \nu}\right), \\
P_{\infty}(\boldsymbol{r}, t ; \varepsilon) & =\xi^{d_{\mathrm{f}}-2 d_{\mathcal{P}}} \mathcal{P}_{\infty}\left(r / \xi, t \xi^{-d_{\mathrm{w}}}\right)
\end{aligned}
$$

where in the new scaling function $\mathcal{P}_{\mathrm{F}}$, we have replaced the cluster size $s$ by the linear extent of $s$-clusters, $R_{s} \sim s^{1 / d_{\mathrm{f}}}$, which serves as reference length scale. Note that $\mathcal{P}_{\mathrm{F}}$ is still analytic in all arguments. For the van Hove functions, this implies a scaling form

$$
\begin{aligned}
G_{s}(\boldsymbol{r}, t ; \varepsilon) & =R_{s}^{-d} \mathcal{G}_{\mathrm{F}}\left(r / R_{s}, t R_{s}^{-d_{\mathrm{w}}}, \varepsilon R_{s}^{1 / \nu}\right), \\
G_{\infty}(\boldsymbol{r}, t ; \varepsilon) & =\xi^{-d} \mathcal{G}_{\infty}\left(r / \xi, t \xi^{-d_{\mathrm{w}}}\right) .
\end{aligned}
$$

The second moments define the cluster-resolved MSDs and possess the scaling forms

$$
\begin{aligned}
\delta r_{s}^{2}(t ; \varepsilon) & =t^{2 / d_{\mathrm{w}}} \delta \mathcal{R}_{\mathrm{F}}^{2}\left(t R_{s}^{-d_{\mathrm{w}}}, \varepsilon R_{s}^{1 / \nu}\right), \\
\delta r_{\infty}^{2}(t ; \varepsilon) & =t^{2 / d_{\mathrm{w}}} \delta \mathcal{R}_{\infty}^{2}\left(t \xi^{-d_{\mathrm{w}}}\right) .
\end{aligned}
$$

Note, that most clusters are much smaller than the correlation length, $R_{s} \ll \xi$, implying $|\varepsilon| R_{s}^{1 / \nu} \ll 1$. For these clusters, $\delta r_{s}^{2}(t ; \varepsilon)$ is insensitive to the distance to the critical point $\varepsilon$, and thus eq. (12a) essentially constitutes a one-parameter scaling prediction, which we will discuss below. The second relation, eq. 12b), is the familiar result for a random walker restricted to the infinite cluster.

We have generated a total of more than 1000 trajectories on 20 square lattices with a linear extent of $L=8,000$ sites for several densities $p$ each. Periodic boundaries were used to minimise finite-size effects, the starting points of the ant were chosen at random, and the size of the clusters was determined using a union-find algorithm [20].

The cluster-resolved MSDs $\delta r_{s}^{2}(t)$ at the threshold are exhibited in fig. 2. all of them follow the same power law $t^{2 / d_{\mathrm{w}}}$ before they saturate. The scaling prediction eq. (12a) implies that the respective long-time limits yield the radii of gyration and grow as $R_{s} \sim s^{1 / d_{\mathrm{f}}}$. The crossover time from subdiffusion to localisation is expected to scale as $t_{\times} \sim R_{s}^{d_{\mathrm{w}}}$. Figure 3 demonstrates that our data exhibit this scaling remarkably well; they bridge the crossover region from anomalous transport to localisation, which extends over three orders of magnitude in time. We have also

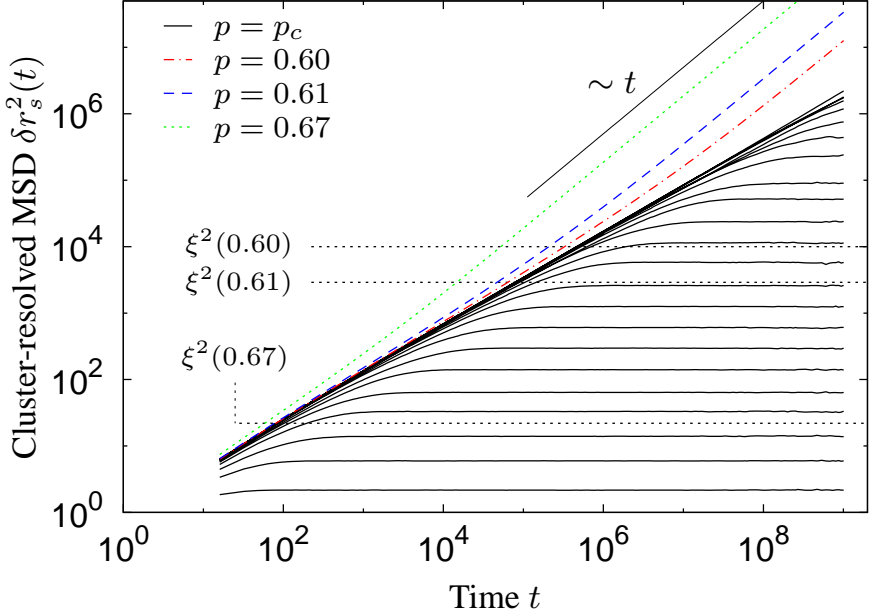

Fig. 2: Cluster-resolved MSDs $\delta r_{s}^{2}(t)$ at the threshold (solid lines); $\delta r_{\infty}^{2}(t)$ is shown for several densities above the threshold. Distances are measured in units of the lattice spacing and time refers to the number of hopping attempts.

included the MSD on the infinite cluster $\delta r_{\infty}^{2}(t)$ above the threshold in fig. 2. The crossover to diffusive transport occurs once the MSD is of the order of $\xi^{2}$, i.e., right at the length scale above which the system looks homogeneous, or in terms of times, $t_{\times} \sim \xi^{d_{\mathrm{w}}}$, in consistency with eq. (12b). The scaling prediction is illustrated in fig. 固 corroborating an earlier study (fig. 6.2 in ref. [5]). In summary, the observed scaling behaviour validates the notion of self-similarity also for the structures of the clusters and the transport on them, provided the system is close to the critical point.

All-cluster-averaged MSD. The all-cluster-averaged MSD $\delta r_{\mathrm{av}}^{2}(t)$ is calculated from the data for the clusterresolved MSDs and the weights $s n_{s}$ and $P$,

$$
\delta r_{\mathrm{av}}^{2}(t ; \varepsilon)=\sum_{s} s n_{s}(\varepsilon) \delta r_{s}^{2}(t)+P(\varepsilon) \delta r_{\infty}^{2}(t)
$$

Using the scaling predictions eqs. (6) and (12), one easily

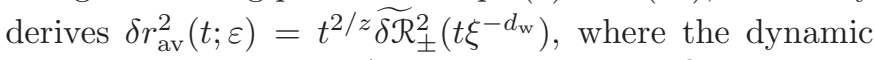
exponent $z$ emerges. At criticality, the infinite cluster has zero weight and the finite clusters contribute an infinite hierarchy of cluster sizes, resulting in an unbounded MSD, diverging as $\delta r_{\text {av }}^{2}(t) \sim t^{2 / z}$. Below the threshold, $\delta r_{s}^{2}(t \rightarrow \infty) \sim R_{s}^{2}$ implies that the long-time limit of the averaged MSD measures the mean-square cluster size, $\delta r_{\mathrm{av}}^{2}(t \rightarrow \infty)=: \ell^{2}$, where $\ell \sim|\varepsilon|^{-\nu+\beta / 2}$. Consistency with the dynamic scaling for $\delta r_{\mathrm{av}}^{2}(t)$ requires $\ell^{z} \sim \xi^{d_{\mathrm{w}}}$, allowing to write the scaling form more transparently,

$$
\delta r_{\mathrm{av}}^{2}(t)=t^{2 / z} \delta \mathcal{R}_{ \pm}^{2}\left(t \ell^{-z}\right) .
$$

On large time scales when $\delta r_{\text {av }}^{2}(t) \gg \xi^{2}$, merely the infinite cluster contributes to the MSD and transport becomes diffusive. With the ansatz $\delta r_{\mathrm{av}}^{2}(t) \sim D t$, eq. (14) dictates that the diffusion coefficient $D$ vanishes at the threshold as $D \sim|\varepsilon|^{\mu}$ where $\mu=\nu\left(d_{\mathrm{w}}-2\right)+\beta$. 


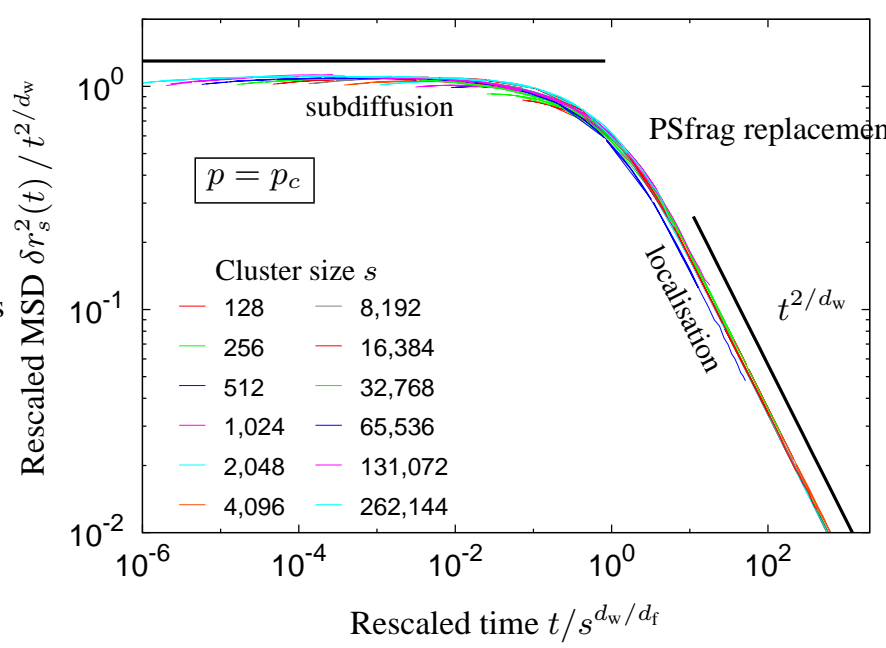

Fig. 3: Cluster-resolved data from fig. 2 rescaled with $s^{1 / d_{\mathrm{f}}}$, which is proportional to the radius of gyration $R_{s}$.

Close to the percolation threshold, our numerical results for the MSD $\delta r_{\mathrm{av}}^{2}(t ; \epsilon)$ follow the critical law, $\delta r_{\mathrm{av}}^{2}(t) \sim$ $t^{2 / z}$, until the curves fan out to either diffusive or localised behaviour (inset of fig. (4). We have rescaled the simulated data according to eq. (14) and find excellent data collapse, see fig. 3. The crossover region covers approximately four nontrivial decades in rescaled time. Let us emphasise that to achieve a data collapse of similar quality for the $3 \mathrm{D}$ Lorentz model, it was necessary to consider explicitly the leading corrections to scaling [4].

Corrections to dynamic scaling. - The leading dynamic corrections to scaling are derived from the scaling form of the joint probabilities for finite and infinite clusters, eqs. (9), along the same lines as for the cluster size distribution. For the MSD on the infinite cluster this implies $\delta r_{\infty}^{2}(t ; \varepsilon)=t^{2 / d_{\mathrm{w}}} \delta \mathrm{R}_{\infty}^{2}\left(t \xi^{-d_{\mathrm{w}}}, u \xi^{-\omega}\right)$. Expanding to first order in the irrelevant scaling variable $u$ yields

$$
\delta r_{\infty}^{2}(t)=t^{2 / d_{\mathrm{w}}} \delta \mathcal{R}_{\infty}^{2}\left(t \xi^{-d_{\mathrm{w}}}\right)\left[1+t^{-y} \Delta_{\infty}\left(t \xi^{-d_{\mathrm{w}}}\right)\right],
$$

where the correction is quantified by the new exponent $y=\omega / d_{\mathrm{w}}$ and a new scaling function $\Delta_{\infty}(\cdot)$. Specifically at the critical point, the cluster-resolved scaling hypothesis predicts $\delta r_{\infty}^{2}(t)=A_{\infty} t^{2 / d_{\mathrm{w}}}\left(1+C_{\infty} t^{-y}\right)$ with the nonuniversal amplitudes $A_{\infty}:=\delta \mathcal{R}_{\infty}^{2}(0)$ and $C_{\infty}:=\Delta_{\infty}(0)$, compare eq. (2). Thus, we have derived the exponent relation, $y d_{\mathrm{w}}=\omega=\Omega d_{\mathrm{f}}$, between the dynamic and static correction exponents $y$ and $\Omega$, mentioned in the introduction.

A similar chain of arguments for the MSD on the finite clusters extends eq. (12a) at criticality to

$$
\delta r_{s}^{2}(t)=t^{2 / d_{\mathrm{w}}} \delta \mathcal{R}_{\mathrm{F}}^{2}\left(t R_{s}^{-d_{\mathrm{w}}}\right)\left[1+t^{-y} \Delta_{\mathrm{F}}\left(t R_{s}^{-d_{\mathrm{w}}}\right)\right] .
$$

Observing that the MSDs on a finite and the infinite cluster are indistinguishable at not too long time scales, $t \ll$ $R_{s}^{d_{\mathrm{w}}}, \xi^{d_{\mathrm{w}}}$, we infer $\delta \mathcal{R}_{\mathrm{F}}^{2}(0)=A_{\infty}$ and $\Delta_{\mathrm{F}}(0)=C_{\infty}$. Calculating the all-cluster average, eq. (13), from eqs. (6), (15),

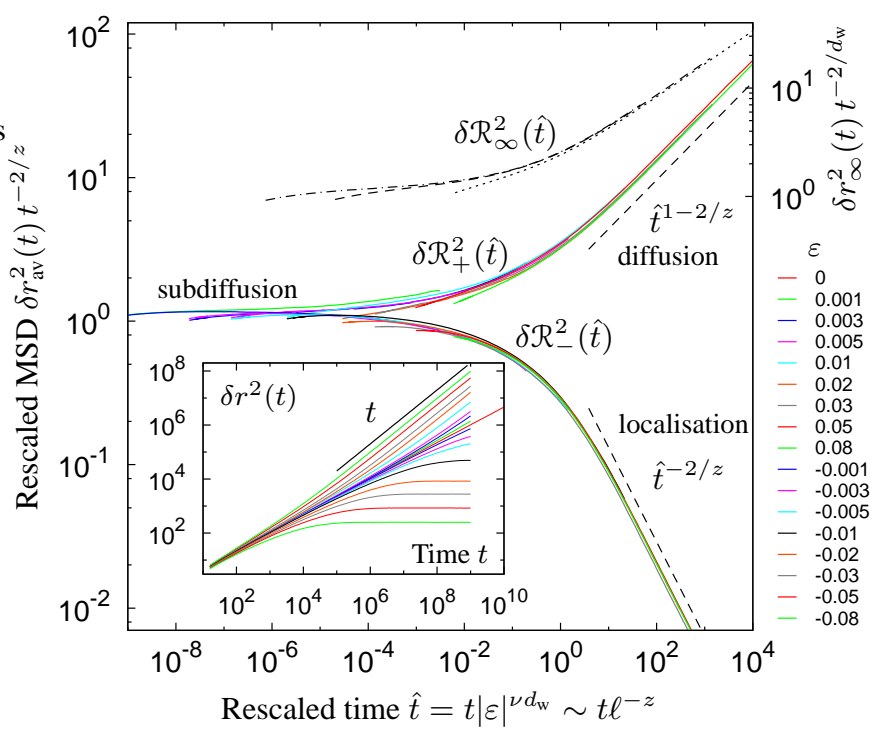

Fig. 4: All-cluster-averaged MSDs $\delta r_{\mathrm{av}}^{2}(t ; \epsilon)$ close to the threshold. While the inset shows the raw data, rescaling according to eq. (14) collapses all data on the scaling functions $\delta \mathcal{R}_{ \pm}^{2}()$. Right axis: rescaled MSDs on the infinite cluster, $\delta r_{\infty}^{2}(t ; \epsilon)$, from fig. 2, following eq. (12b).

(16) leads to

$$
\delta r_{\mathrm{av}}^{2}(t)=t^{2 / z} \delta \mathcal{R}_{ \pm}^{2}\left(t \ell^{-z}\right)\left[1+t^{-y} \Delta_{ \pm}\left(t \ell^{-z}\right)\right],
$$

where the scaling variable can equivalently be written as $t \xi^{-d_{\mathrm{w}}} \propto t \ell^{-z}$. We have suggested such a form already for the 3D Lorentz model [4], and here we provide the basis for this ansatz. In particular, we shall test the prediction at the critical point,

$$
\delta r_{\mathrm{av}}^{2}(t) \simeq A_{\mathrm{av}} t^{2 / z}\left(1+C_{\mathrm{av}} t^{-y}\right) \quad \text { for } \quad t \rightarrow \infty .
$$

For a numerical analysis of the dynamic corrections to scaling, we have simulated 4,070 trajectories of $10^{11}$ steps on periodic square lattices with a linear extent of $L=$ 90,000 sites at the critical density 2 the starting points were chosen at random on arbitrary clusters. To increase computational efficiency, we utilised the statistical independence on the bit-level of the random number generator GFSR4 [21]: calculating one random 32-bit integer allowed the realisation of 16 hopping attempts. Figure 5 shows the rectified MSD $\delta r_{\mathrm{av}}^{2}(t) / A_{\mathrm{av}} t^{2 / z}$ at the threshold; a fit for the amplitude yields $A_{\mathrm{av}}=1.204(1)$. The leading asymptotic behaviour is very well captured over 6 decades in time with the exponent $z$ obtained from conductivity measurements, see table 1 On a double-logarithmic scale, the deviations from the leading behaviour are compatible with a power law decay with exponent $y=0.49$. There is still a relatively large, overall statistical error in our data of about $1 \%$ since the heterogeneous average over clusters of all sizes converges quite slowly. Better convergence is

\footnotetext{
${ }^{2}$ Each trajectory required a computing time of about 19 hours on a single core of the AMD Opteron $82182.6 \mathrm{GHz}$ processor.
} 


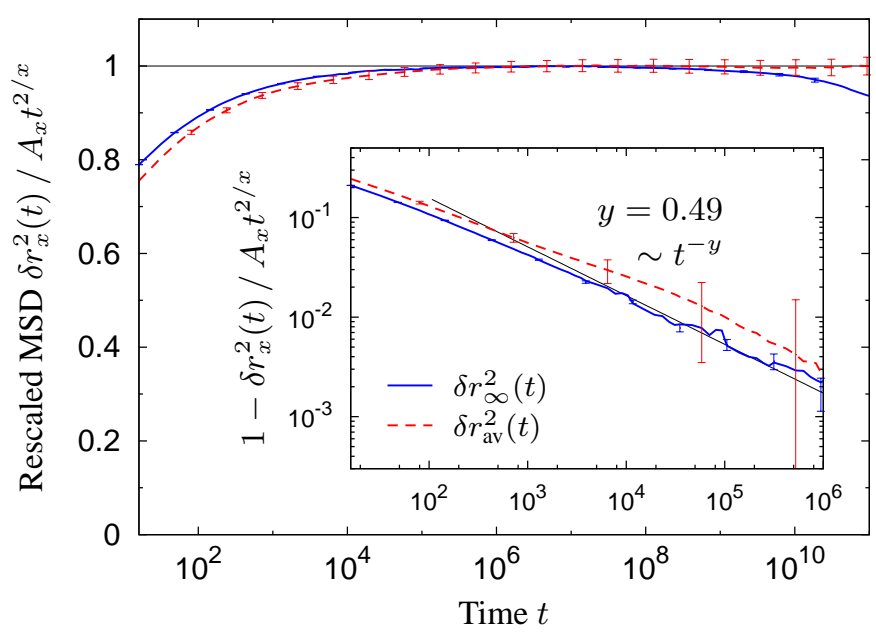

Fig. 5: Rectified MSDs restricted to the infinite cluster (solid, blue) and averaged over all clusters (broken, red) at the critical density. Data for $\delta r_{\infty}^{2}(t)$ are rescaled with $A_{\infty} t^{2 / d_{\mathrm{w}}}$ and for $\delta r_{\text {av }}^{2}(t)$ with $A_{\mathrm{av}} t^{2 / z}$. Inset: a double-logarithmic plot of the relative deviations from the asymptotic behaviour shows that the leading corrections indeed follow the predicted power law.

expected if the random walkers are restricted to the infinite cluster alone. We have generated 2,266 trajectories at criticality on the largest cluster of periodic lattices with $L=13,500$, which allowed a measurement of $\delta r_{\infty}^{2}(t)$ with a precision of $1 \%$ for $t \lesssim 10^{8}$ steps. Rescaled with the asymptotic law $A_{\infty} t^{2 / d_{\mathrm{w}}}$ where $A_{\infty}=1.152(3)$, the data converge to a plateau within $3 \%$ o for a decade in time; for times longer than $t \gtrsim 10^{7}$, the data start to deviate from the plateau, we have checked that this is a finite-size effect. Nevertheless, the approach to the plateau at shorter time scales is well captured with our predicted power law, $t^{-y}$ with $y=0.49$, over two decades in time, see inset of

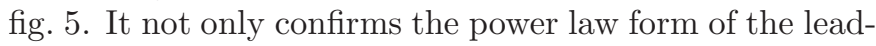
ing corrections to scaling, but also the derived exponent relation between $\Omega$ and $y$, eq. (3).

Conclusions. - We have derived an exponent relation connecting the leading corrections to scaling of the static cluster structure and the transport dynamics. The derivation relies on a cluster-resolved scaling theory unifying structure and dynamics, which has been corroborated in detail by simulations for the square lattice.

The corrections to scaling discussed here have their origin in the same leading irrelevant scaling field $u$ for the statics and dynamics. Yet, the motion of a random walker on the fractal clusters introduces new, genuinely dynamic scaling fields, one relevant and infinitely many irrelevant ones. In our approach, we have anticipated that the leading irrelevant scaling field is still provided by the static field $u$, in agreement with our simulations. Analytic deformations of the scaling fields typically lead to analytic corrections with integral relative exponents [12]. Here, we find $\Omega, y<1$ and conclude that the universal corrections dominate over the analytic contributions.
Finally, our analysis suggests to interpret experiments on anomalous diffusion in disordered environments in terms of universal exponents, including leading corrections to the asymptotic behaviour, rather than fitting with phenomenological exponents. Percolation provides one possible universality class for such an approach.

$$
* * *
$$

We thank John Cardy and Erwin Frey for valuable discussions on the corrections to scaling in the renormalisation group. Financial support by the Nanosystems Initiative Munich (NIM) is gratefully acknowledged.

\section{REFERENCES}

[1] Golding I. and Cox E. C., Phys. Rev. Lett., 96 (2006) 098102; Weiss M., Elsner M., Kartberg F. and NilsSON T., Biophys. J., 87 (2004) 3518.

[2] Nicolau, Dan V. J., Hancock J. F. and Burrage K., Biophys. J., 92 (2007) 1975.

[3] Sung B. J. and Yethiraj A., Phys. Rev. Lett., 96 (2006) 228103; Saxton M. J., Biophys. J., 66 (1994) 394.

[4] Höfling F., Franosch T. and Frey E., Phys. Rev. Lett., 96 (2006) 165901; HöFling F., Munk T., Frey E. and Franosch T., J. Chem. Phys., 128 (2008) 164517.

[5] Ben Avraham D. and Havlin S., Diffusion and Reactions in Fractals and Disordered Systems (Cambridge University Press) 2000.

[6] Stauffer D. and Aharony A., Introduction to Percolation Theory (Taylor \& Francis, London) 1994.

[7] Grassberger P., Physica A, 262 (1998) 251.

[8] Lorenz C. D. and Ziff R. M., Phys. Rev. E, 57 (1998) 230.

[9] Jan N. and Stauffer D., Int. J. Mod. Phys. C, 9 (1998) 341.

[10] Machta J. and Moore S. M., Phys. Rev. A, 32 (1985) 3164; Halperin B. I., Feng S. and Sen P. N., Phys. Rev. Lett., 54 (1985) 2391.

[11] Höfling F. and Franosch T., Phys. Rev. Lett., 98 (2007) 140601

[12] CARdy J., Scaling and Renormalization in Statistical Physics (Cambridge University Press) 1996.

[13] Margolina A., Nakanishi H., Stauffer D. and StanLEY H. E., J. Phys. A, 17 (1984) 1683.

[14] Ziff R. M., Phys. Rev. Lett., 69 (1992) 2670; NEwman M. E. J. and Ziff R. M., Phys. Rev. Lett., 85 (2000) 4104.

[15] Hoshen J. and Kopelman R., Phys. Rev. B, 14 (1976) 3438.

[16] Rapaport D. C., J. Phys. A, 18 (1985) L175.

[17] Rapaport D. C., J. Phys. A, 19 (1986) 291.

[18] Ben-Avraham D. and Havlin S., J. Phys. A, 15 (1982) L691; Gefen Y., Aharony A. and Alexander S., Phys. Rev. Lett., 50 (1983) 77.

[19] Höfling F., Ph.D. thesis Ludwig-MaximiliansUniversität München, ISBN 978-3-86582-426-4 (2006).

[20] Newman M. E. J. and Ziff R. M., Phys. Rev. E, 64 (2001) 016706.

[21] Ziff R. M., Comp. Phys., 12 (1998) 385. 